\title{
Discrimination ability of the endotracheal tube location using real-time palpation during intubation using an endotracheal tube with a preloaded stylet
}

This article was published in the following Dove Press journal:

Therapeutics and Clinical Risk Management

\author{
Go Un Roh' \\ Yun Jeong Chae ${ }^{2}$ \\ Young Bok Lee ${ }^{3}$ \\ Wikwang Wang ${ }^{3}$ \\ Chang Ik Choi ${ }^{3}$ \\ In Kyong $\mathrm{Yi}^{2}$
}

'Department of Anesthesiology and Pain Medicine, College of Medicine, CHA University, Bundang CHA General Hospital, Seongnam,

South Korea; ${ }^{2}$ Department of Anesthesiology and Pain Medicine, Ajou University School of Medicine, Suwon, South Korea; ${ }^{3}$ Department of Anesthesiology and Pain Medicine, Yonsei University, Wonju College of Medicine, Wonju, South Korea
Correspondence: In Kyong Yi

Department of Anaesthesiology and Pain Medicine, Ajou University School of Medicine 164, Worldcup-ro, Yeongtong-gu, Suwon 16499,

South Korea

Tel +82312197522

Fax +8231219579

Email lyrin0।@gmail.com
Purpose: Palpation during intubation could be used as an ancillary method of providing real-time information of the endotracheal tube (ETT) placement before manual ventilation. This study aimed to evaluate the ability to discriminate the ETT location using a modified real-time palpation method with a preloaded stylet during intubation.

Patients and methods: The examiner performing the real-time palpation method placed three fingers on the lateral sides of the trachea between the sternal notch and the thyroid cartilage to determine if endotracheal intubation was successful. Endotracheal intubation was confirmed by auscultation and quantitative carbon dioxide waveform using capnography.

Results: Eighty-eight patients were enrolled in this study. The discrimination accuracy of the real-time palpation method was $98.9 \%$ (95\% CI: 93.8-99.8) for identifying the location of ETT between the trachea and esophagus. There was one false negative, reported as esophageal intubation rather than tracheal intubation.

Conclusion: The real-time palpation method during intubation using an ETT with a preloaded stylet is an instantly applicable technique with good discrimination ability. The addition of realtime information through this palpation method to the conventional intubation process, especially in patients with poor vocal cord visualization, would be useful to facilitate the process of safe and careful endotracheal intubation.

Keywords: airway management, endotracheal, esophageal, intubation, palpation

\section{Introduction}

Verification of endotracheal tube (ETT) placement is a vital process because a delay in recognizing esophageal intubation could lead to potentially fatal complications. ${ }^{1}$ Therefore, all available methods should be mobilized and include fail-safe or almost fail-safe methods such as direct visualization of the vocal cords, quantitative carbon dioxide waveform using capnography, and fiberoptic bronchoscopic inspection of the tracheal ring and carina. ${ }^{2}$ Therefore, the palpation method cannot be substituted for these aforementioned fail-safe methods. Instead, it has clinical value as a complementary means of providing real-time information of ETT placement. It is particularly applicable when it is hard to see the vocal cords in patients with high Cormack and Lehane scores or an airway view obscured by blood or secretions. ${ }^{3,4}$ The active use of palpation in several clinical situations could still be regarded as useful. Real-time information could reduce the excessive effort often placed on exposing the vocal cords and the number of intubation attempts around the entrance to the glottis. 
Subsequently, employing this method could reduce trauma during intubation. In addition, it could lead to confident and careful verification of ETT placement. Thus, it allows the physician to pay attention to gastric inflation especially when esophageal intubation is suspected. It is also useful for training purposes, allowing a supervising anesthesiologist to verify if the trainee has positioned the tube correctly and then provide direct feedback.

Various palpation methods for determining the ETT location have been proposed. ${ }^{3}$ In particular, the concept of using palpation with the fingers over the trachea during intubation has been studied. ${ }^{5-7}$ Contrary to the results in infants, ${ }^{6}$ the accuracy of this method in adults was found to be unsatisfactory. ${ }^{5,7}$ In a study on infants, ${ }^{6}$ in addition to the thin skin present, the use of a stylet may strengthen the sensation felt during palpation, allowing better discrimination. Although it has not been used in adult research, the use of a stylet in clinical situations where intubation is difficult is essential. The preloaded stylet could increase the sensation felt during palpation, more so than the ETT itself. While advancing the ETT away from the stylet, the tip of the ETT is angled more anteriorly, allowing it to collide with the anterior wall of the trachea more strongly. ${ }^{8}$ Therefore, the discrimination ability of palpation may be better than the previously reported result. Moreover, we assumed that holding the lateral side of the trachea between the sternal notch and cricoid cartilage might be effective for determining the location of the ETT through strengthened tactile sensation between the fingers. In other words, a palpation method that incorporates a preloaded stylet into an ETT in addition to placement of the fingers for palpation and sensation during intubation might be useful as a real-time ancillary technique to discriminate between tracheal and esophageal intubation.

The purpose of this study was to evaluate the ability of this modified real-time palpation method using a preloaded intubating stylet to discriminate the ETT location during intubation in adults undergoing elective surgery.

\section{Patients and methods}

After obtaining approval from the Institutional Review Board of Ajou University Hospital (AJIRB-MED-OBS-14-379), this study was registered at https://cris.nih.go.kr/cris (Identifier number: KCT0001387) and conformed to the tenets of the Declaration of Helsinki. Patients with an American Society of Anesthesiologists physical status I or II, aged 20-60 years, undergoing general surgery, such as laparoscopic cholecystectomy, breast, inguinal hernia, or ophthalmic surgery, requiring general anesthesia with endotracheal intubation were included after obtaining written informed consent. Patients were excluded if they had a history of difficult intubation, poor dentition, gastroesophageal reflux, obesity (body mass index $>35 \mathrm{~kg} / \mathrm{m}^{2}$ ), or any anatomical abnormality of the face or neck. The stylet we used was malleable aluminum stylet wrapped with clear polyvinyl chloride (Intubating stylet, SL1P10, Hangzhou Shanyou Medical Equipment Co., Ltd, China).

On arrival to the operating room, the patients were monitored with electrocardiography, continuous pulse oximetry, and noninvasive measurement of blood pressure. Patients were placed in a neutral position with slight elevation of the head to $5 \mathrm{~cm}$ using a pillow. Anesthesia was induced with thiopental $(5 \mathrm{mg} / \mathrm{kg}$ ) or propofol $(2 \mathrm{mg} / \mathrm{kg})$ and rocuronium $(0.6 \mathrm{mg} / \mathrm{kg})$ at the anesthesiologist's discretion. After manual ventilation with $100 \%$ oxygen for 2 minutes, endotracheal intubation was performed using a 7.0-mm ETT for female and 8.0-mm ETT for male patients. The stylet was preloaded inside the ETT, and subsequently bent into a hockey stick shape with a $45^{\circ}$ angle. The tip of the stylet was positioned proximal to the Murphy eye. The anesthesiologists performing the intubation were junior anesthesia residents who were asked to advance the ETT off the stylet in one try without any airway optimal maneuver or repositioning of the patients, so as not to give the examiner any clues regarding the intubation. The examiner performing the real-time palpation method placed three fingers in close contact on the lateral sides of the trachea between the sternal notch and the thyroid cartilage: the thumb was placed on one side and the index and middle fingers on the other side of the trachea. The examiner ensured that her thumb was not touching her index and middle fingers. The examiner then closed her eyes, turned her head toward the patient's foot, and judged whether it was an endotracheal or esophageal intubation. When there was a tactile sensation in the space between the fingertips, it was judged as an endotracheal intubation, regardless of the characteristics of the sensation. On the other hand, if there was no sensation detected between the fingertips, it was judged as an esophageal intubation. The same examiner performed the palpation process in all patients. The examiner had performed this procedure 10 times previously, had no formal training, and had no experience of palpation in esophageal intubation.

Endotracheal intubation was confirmed by auscultation and detection of exhaled carbon dioxide $(>4 \mathrm{mmHg}$ after at least five breaths) with a typical waveform on capnography. ${ }^{9}$ If esophageal intubation was confirmed, reintubation was performed. 
A sample size calculation was performed using the area under the receiver-operating characteristic curve (AUC), which provides consistent accuracy with binary data. ${ }^{10}$ We assumed that the AUC of the real-time palpable method using the preloaded stylet to predict the success of endotracheal intubation was at least 0.8 , which is considered as excellent discrimination, given an alpha error of 0.05 and a power of $90 \%{ }^{11,12}$ Using a prevalence of esophageal intubation of $10 \%$, the required sample size was 88 . Statistical analysis was performed using the SPSS 20.0 statistical package (SPSS Inc., Chicago, IL, USA) and MedCalc software version 11.0.1 (MedCalc Software, Mariakerke, Belgium). The AUC, sensitivity, specificity, positive and negative predictive values, accuracy, and the $95 \% \mathrm{CI}$ were calculated. If the lower limit of the $95 \%$ CI for AUC was $>0.8$, the real-time palpable method was considered to have a clinically useful discriminatory potential. Comparison between tracheal and esophageal intubation was performed using a Student's $t$-test or chi-squared test as appropriate.

\section{Results}

A total of 88 patients who met the inclusion criteria consented to participate in this study. The patient characteristics are presented in Table 1. There were no significant differences between patient characteristics in the tracheal and esophageal intubation groups.

The test results of the real-time palpation method are shown in Table 2. The calculated AUC and accuracy of the real-time palpation were 0.994 (95\% CI: 0.979-1.000) and 98.9\% (95\% CI: 93.8-99.8), respectively, for discriminating between tracheal and esophageal intubation, indicating that the real-time palpation method had excellent discrimination accuracy. ${ }^{13}$ The overall sensitivity and specificity of the realtime palpation method were $98.8 \%$ (95\% CI: 93.2-100) and $100 \%$ (95\% CI: 63.1-100), respectively. There was one false negative, reported as an esophageal intubation through the palpation method, which capnography later revealed to be a tracheal intubation.

Table I Demographic characteristics of patients

\begin{tabular}{lll}
\hline & $\begin{array}{l}\text { Tracheal } \\
\text { intubation }\end{array}$ & $\begin{array}{l}\text { Esophageal } \\
\text { intubation }\end{array}$ \\
\hline Age (years) & $46.3 \pm 10.4$ & $38.9 \pm 1 \mathrm{I} .2$ \\
$\mathrm{BMI}\left(\mathrm{kg} / \mathrm{m}^{2}\right)$ & $23.5 \pm 3.0$ & $24.9 \pm 3.1$ \\
Sex $(\mathrm{M} / \mathrm{F})$ & $29 / 5 \mathrm{I}$ & $5 / 3$ \\
ASA PS (I/II) & $60 / 20$ & $7 / 1$ \\
\hline
\end{tabular}

Note: Values are mean \pm SD or number of patients.

Abbreviations: ASA PS, American Society of Anesthesiologists physical status; $B M I$, body mass index; M/F, male/female.
Table 2 Test results using palpation to distinguish tracheal and esophageal intubation

\begin{tabular}{llll}
\hline & $\begin{array}{l}\text { Tracheal } \\
\text { intubation }\end{array}$ & $\begin{array}{l}\text { Esophageal } \\
\text { intubation }\end{array}$ & Total \\
\hline Real-time palpation, tracheal & $79(89.8)$ & $0(0.0)$ & $79(89.8)$ \\
Real-time palpation, esophageal & $\mathrm{I}(\mathrm{I} . \mathrm{I})$ & $8(9.1)$ & $9(10.2)$ \\
Total & $80(90.9)$ & $8(9.1)$ & $88(100)$ \\
\hline
\end{tabular}

Note: Values are number of patients (\%).

\section{Discussion}

This study evaluated the ability of a modified real-time palpation method using an ETT with a preloaded stylet to discriminate the ETT location. The palpation method had good discrimination accuracy (98.9\%, 95\% CI: 93.8-99.8) in its ability to differentiate between endotracheal and esophageal intubation and both high sensitivity and specificity.

To date, there have been several reports on palpation techniques for the confirmation of endotracheal intubation because of its noninvasive and instantly applicable characteristics. ${ }^{3}$ A study by Cameron et al evaluated standard cricoid pressure and suprasternal pressure over the first and second tracheal rings in 20 adults, respectively. Their study showed that the method using cricoid rather than suprasternal pressure was slightly better. However, four out of ten esophageal intubations could not be differentiated, which precluded them from recommending the palpation method for clinical application. ${ }^{5}$ This finding was in contrast to the study by Bednarek and Kuhns in which palpation at the level of the clavicle could correctly identify the location of the ETT in most cases of deceased infants. ${ }^{6}$ We assumed that the difference in results between the two studies might be from the difference between the tactile strength of the skin. The skin of an infant is much thinner than that of an adult. In addition, a stylet was used in the study with infants and not in the previous study in adults. ${ }^{6}$ These factors may have strengthened the sensation during palpation and seemed to enhance the discrimination ability. Indeed, a study by Bair et a ${ }^{14}$ supports this reasoning. Although their study was different for several reasons (they used adult cadavers and a gum elastic bougie, and performed palpation after intubation), they demonstrated that tactile perception over the tracheal rings using the angled tip of a bougie was both very sensitive and specific for differentiating between tracheal and esophageal intubation even in adults. ${ }^{14}$ This principle of tactile perception was applied in the present study. The stylet was preloaded inside the ETT, and subsequently bent into a hockey stick shape. Consequently, the tip of the ETT is directed at a more anterior angle while the anteriorly bent stylet is withdrawn, 
so it tends to collide with the tracheal rings strongly while advancing the ETT away from the stylet. ${ }^{8,15}$ Therefore, this stronger sensation could be more easily palpated than that of the naturally curved ETT itself, which was used in the study by Cameron et al. In the present study, the modified real-time palpation method with a preloaded stylet was superior to that by Cameron et al, as the discrimination accuracy was $98.9 \%$ vs $70 \%-80 \%$, respectively. ${ }^{5}$

Another characteristic of the palpation method in the present study is the modified tactile method for perception. In previous reports, the fingers were usually applied horizontally on the anterior surface of the tracheal ring and results focused on whether there was a specific tactile sensation felt, such as a corrugated sensation. ${ }^{6,7,14}$ Therefore, in cases with weak and short palpable sensations without a specific tactile character, it might be difficult to discriminate between tracheal and esophageal intubation. In this study, three fingers were used to hold the lateral surface of the tracheal ring, making it easy to detect the stronger sensation during ETT advancement into the trachea. In addition, according to the examiner, in some cases of tracheal intubation, the characteristic of the sensation was not corrugated, but instead there was a sustained nontypical sensation located between the fingers. In contrast, during cases of esophageal intubation, the sensation detected was not between the fingers or there was nothing felt. Therefore, a stronger sensation, even if there was no typical texture felt, seemed to be more discriminative while using the tactile method at the lateral surface of the tracheal rings.

The use of a stylet predisposes to tracheal trauma. ${ }^{16}$ Trauma can be caused by contact of the stylet tip to the subglottic structure or the brittle broken pieces of the stylet. In addition, the hockey stick-shaped curvature of an ETT may make the tip of an ETT induce injury to the anterior part of the trachea while withdrawing the stylet. ${ }^{17}$ To reduce these risks, we took care to position the tip of stylet inside the lumen of an ETT, limit the angle of a preloaded stylet into an ETT to a $45^{\circ}$ angle, and use a disposable stylet wrapped with polyvinyl chloride. Once the tip of the ETT has passed the vocal cords, the stylet was withdrawn as the ETT was advanced. ${ }^{18,19}$ In addition to these, the type of stylet and the force to advance ETTs can affect the risk of trauma. Various types of stylets are available, including the standard malleable stylet, the rigid stylet (eg, GlideRite Rigid Stylet), the Parker Flex-It articulating stylet, and the gum elastic bougie. The incidence of trauma is lower with the malleable stylet or Parker Flex-It stylet when compared to the rigid stylet. ${ }^{20,21}$ In addition, ETTs should be inserted with minimal force and the bending angle rechecked when resistance is encountered during inserting the ETT into the trachea. ${ }^{20,22}$ Above all, clinicians should consider the risks and benefits of the real-time palpation method using a preloaded stylet rather than the routine use. We think that indications for the real-time palpation would be similar to that for the stylet. Based on our clinical experience, both the stylet and the real-time palpation method are expected to be useful in clinical situations where direct vocal cord visualization is difficult, for example, in patients with a high Cormack and Lehane score, or an obstructed airway from blood or secretions. ${ }^{3,4}$ Under these situations, it is a high priority to secure an airway using the stylet in a quick manner and through a minimal number of attempts, to avoid further airway trauma. Thus, in a clinical situation requiring the use of a stylet, it would be useful to actively utilize the palpation method. The use of real-time information could assist the physician with reducing the excess in effort required to expose the vocal cords and the number of intubation attempts, which can often result in trauma and bleeding to the surrounding tissue. In addition, it could lead to an increase in physician focus and attention for verifying ETT placement. If palpation is utilized for endotracheal intubation, the physician could proceed immediately to the next verification process with confidence, whereas if it is an esophageal intubation, he/she could pay special attention not to cause excessive gastric inflation, which can predispose a patient to vomiting and regurgitation ${ }^{23}$ during the verification process.

In this study, the incidence of esophageal intubation was $9.1 \%$. We assessed tracheal intubation and esophageal intubation based on first-attempt intubation. In all cases of esophageal intubation, success was achieved in the second attempt. The junior residents performed the intubation in a single attempt without airway optimal maneuver or repositioning of the patients. Therefore, the training level of the junior residents and the assessment of first-attempt intubation without any optimal maneuver might have affected the incidence of esophageal intubation in our study. This is slightly less than that reported in the previous reports, in which the failure rate of endotracheal intubation in the first attempt was $19.8 \%-20.0 \% .^{24,25}$

The various methods for confirmation of ETT location can be categorized into non-fail-safe, almost fail-safe, and fail-safe. ${ }^{2}$ The palpation method could be classified as a nonfail-safe method because its information depends intrinsically on the tactile sensation and the CI of specificity in this study was still broad. Confirmation of endotracheal intubation is an essential and life-saving step during airway management. ${ }^{26,27}$ 
Therefore, the palpation method should not be substituted for fail-safe or almost fail-safe methods, such as direct visualization of vocal cords, quantitative carbon dioxide waveform on capnography, and fiberoptic bronchoscopic inspection of the tracheal ring and carina. Instead, the real-time palpation method during intubation has major implications to prevent such consequences. In addition, this method could be instantly used as one of the multiple non-fail-safe methods to increase the accuracy of the confirmation of ETT location when essential fail-safe methods are limited.

This study has several limitations. First, there were only eight cases of esophageal intubation (9.1\%). However, this study would be improved if we could validate the specificity of the palpation method with more cases of esophageal intubation, given that the CIs for specificity in the present study were broad. Secondly, only one anesthesiologist with experience using this method performed the palpation technique. Experience could affect the accuracy of results to some extent. ${ }^{14}$ In this study, the examiner had only palpated endotracheal intubation on ten occasions and had no experience at all with palpating esophageal intubation. Nevertheless, the examiner discriminated well. This implies that the palpation method does not require much skill, so the impact of experience on outcome may be small. Thirdly, this method would be difficult to apply in cases where it is difficult to palpate the trachea such as in patients who are obese. Fourth, this method was performed in a refined operating room, not in an emergency setting and in selected patients excluding patients with risk factors associated with a difficult airway. However, we believe that sensation at the level of the trachea is not influenced by a difficult airway at the level of the vocal cords as long as the trachea can be palpated. Fifth, there was one false negative. The examiner described that nothing could be felt at all, so it was considered to be esophageal intubation. A possible explanation for this is that there was weak contact of the tube alongside the trachea. This was unlike the cases of true esophageal intubation, where the examiner reported feeling a sensation at a different location, that is, not directly between the fingers. This implies that the palpation method might be more useful if only certain tactile positions are used.

\section{Conclusion}

The real-time palpation method using an ETT with a preloaded stylet during intubation is an instantly applicable technique with good discrimination ability. The addition of this palpation method to the conventional intubation process, especially in cases of difficult intubation, would be useful to facilitate the process of endotracheal intubation through giving the physician real-time information of the location of the ETT before bagging. This would reduce the side effects caused by excessive intubation attempts and ensure that clinicians are more attentive to the verification process. It could be instantly applied as one of the multiple non-failsafe methods to increase the accuracy for confirmation of the ETT location when essential fail-safe methods are limited. However, to evaluate the usefulness of this technique as a confirmative method, further research is needed.

\section{Acknowledgment}

We would like to thank Editage (www.editage.co.kr) for English language editing.

\section{Disclosure}

The authors report no conflicts of interest in this work.

\section{References}

1. Holland R, Webb RK, Runciman WB. The Australian Incident Monitoring Study. Oesophageal intubation: an analysis of 2000 incident reports. Anaesth Intensive Care. 1993;21(5):608-610.

2. Salem MR, Baraka AS. Confirmation of endotracheal intubation. In: Hagberg CA, editor. Benumof and Hagberg's Airway Management. 3rd ed. Philadelphia, PA: Elsevier/Saunders; 2013:657-682.

3. Rudraraju P, Eisen LA. Confirmation of endotracheal tube position: a narrative review. J Intensive Care Med. 2009;24(5):283-292.

4. Hosseini JS, Talebian MT, Ghafari MH, Eslami V. Secondary confirmation of endotracheal tube position by diaphragm motion in right subcostal ultrasound view. Int J Crit Illn Inj Sci. 2013;3(2):113-117.

5. Cameron AE, Hyde RA, Sivalingam P, Asbury AJ. Detection of accidental oesophageal intubation. Role of the anaesthetic assistant. Anaesthesia. 1997;52(8):733-735.

6. Bednarek FJ, Kuhns LR. Endotracheal tube placement in infants determined by suprasternal palpation: a new technique. Pediatrics. 1975;56(2): 224-229.

7. Ishigaki M, Takahashi S, Tanaka M, Miyabe M. Reliability in detection of tracheal intubation using cricoid palpation method. Masui. 2011; 60(2):138-141.

8. Dupanović M, Isaacson SA, Borovcanin Z, et al. Clinical comparison of two stylet angles for orotracheal intubation with the GlideScope video laryngoscope. J Clin Anesth. 2010;22(5):352-359.

9. Chou HC, Chong KM, Sim SS, et al. Real-time tracheal ultrasonography for confirmation of endotracheal tube placement during cardiopulmonary resuscitation. Resuscitation. 2013;84(12):1708-1712.

10. Huang J, Ling CX. Using AUC and accuracy in evaluating learning algorithms. IEEE Trans Knowl Data Eng. 2005;17(3):299-310.

11. Hanley JA, Mcneil BJ. The meaning and use of the area under a receiver operating characteristic (ROC) curve. Radiology. 1982;143(1): 29-36.

12. Hosmer DW, Lemeshow S. Applied Logistic Regression. 2nd ed. New York, NY: John Wiley \& Sons; 2000.

13. Simundić AM, Tocnost D. Diagnostic accuracy. Acta Med Croatica. 2006;60 Suppl 1:93-111.

14. Bair AE, Laurin EG, Schmitt BJ. An assessment of a tracheal tube introducer as an endotracheal tube placement confirmation device. Am J Emerg Med. 2005;23(6):754-758.

15. Grape S, Schoettker P. The role of tracheal tube introducers and stylets in current airway management. J Clin Monit Comput. 2017; 31(3):531-537. 
16. Divatia JV, Bhowmick K. Complications of endotracheal intubation and other airway management procedures. Indian J Anaesth. 2005; 49(4):308-318.

17. Kundra P, Nagappa M, Elakkumanan LB, Gupta K. A novel technique to avoid tracheal injury during intubation with stylet and flexometallic endotracheal tube. J Anaesthesiol Clin Pharmacol. 2010;26(2):275.

18. Scott J, Hung OR [webpage on the Internet]. Intubating Introducers, Stylets, and Lighted Stylets (Lightwands). iKNOLEGDE. Available from: https://clinicalgate.com/intubating-introducers-stylets-andlighted-stylets-lightwands/. Accessed May 30, 2018.

19. The Regents of the University of California [webpage on the Internet]. Stylets. The Regents of the University of California. Available from: http://aam.ucsf.edu/article/stylets. Accessed May 30, 2018.

20. Sheta SA, Abdelhalim AA, Elzoughari IA, Alzahrani TA, Al-Saeed AH. Parker Flex-It stylet is as effective as GlideRite Rigid stylet for orotracheal intubation by Glidescope. Saudi Med J. 2015;36(12): 1446-1452.

21. Shamim R, Sindwani G, Priya V, Suri A. Saviour of stylet injuries: 'Ryle's tube'. Indian J Anaesth. 2018;62(5):399-400.

22. Levitan RM, Pisaturo JT, Kinkle WC, Butler K, Everett WW. Stylet bend angles and tracheal tube passage using a straight-to-cuff shape. Acad Emerg Med. 2006;13(12):1255-1258.
23. Wenzel V, Idris AH, Dörges $\mathrm{V}$, et al. The respiratory system during resuscitation: a review of the history, risk of infection during assisted ventilation, respiratory mechanics, and ventilation strategies for patients with an unprotected airway. Resuscitation. 2001;49(2):123-134.

24. Ibinson JW, Ezaru CS, Cormican DS, Mangione MP. GlideScope use improves intubation success rates: an observational study using propensity score matching. BMC Anesthesiol. 2014;14:101.

25. Ferrando C, Aguilar G, Belda FJ. Comparison of the laryngeal view during tracheal intubation using Airtraq and Macintosh laryngoscopes by unskillful anesthesiology residents: a clinical study. Anesthesiol Res Pract. 2011;2011:301057.

26. Mark LJ, Herzer KR, Cover R, et al. Difficult airway response team: a novel quality improvement program for managing hospital-wide airway emergencies. Anesth Analg. 2015;121(1):127-139.

27. Grmec S. Comparison of three different methods to confirm tracheal tube placement in emergency intubation. Intensive Care Med. 2002;28(6): 701-704.
Therapeutics and Clinical Risk Management

\section{Publish your work in this journal}

Therapeutics and Clinical Risk Management is an international, peerreviewed journal of clinical therapeutics and risk management, focusing on concise rapid reporting of clinical studies in all therapeutic areas, outcomes, safety, and programs for the effective, safe, and sustained use of medicines. This journal is indexed on PubMed Central, CAS,

\section{Dovepress}

EMBase, Scopus and the Elsevier Bibliographic databases. The manuscript management system is completely online and includes a very quick and fair peer-review system, which is all easy to use. Visit http://www.dovepress.com/testimonials.php to read real quotes from published authors. 